pituitary-thyroid axis no longer functions as an intact unit in such patients, since the irradiated thyroid may be unable to respond to endogenous $\mathrm{TSH}$ by increasing thyroid hormone secretion. The raised serum TSH concentration may simply indicate inadequate amounts of circulating thyroid hormones.

A further argument for giving thyroxine replacement therapy to euthyroid patients with raised serum TSH concentrations after iodine-131 is the possibility that such patients if left untreated might be at increased risk of ischaemic heart disease, as occurs in autoimmune thyroiditis. ${ }^{11}$ Such a decision would be justified, however, only if the association between autoimmune thyroiditis and ischaemic heart disease, which is at present unknown, was to be mediated via raised serum TSH and suboptimal thyroid hormone concentrations rather than other possible factors such as hypercholesterolaemia ${ }^{12}$ and circulating immune complexes. ${ }^{13}$ If thyroxine is to be administered to clinically euthyroid patients with raised serum TSH concentrations it must be given with caution. About half of all patients treated with iodine-131 for thyrotoxicosis have circulating TSH receptor antibodies, ${ }^{14}$ and thus the thyroid remnant, although unable to sustain normal output of thyroid hormones, may be functioning autonomously and not under the control of endogenous TSH. Thyroxine therapy would be additive to the remnant production of thyroid hormones in some patients, and if the average replacement of $0 \cdot 15 \mathrm{mg}$ daily was to be given ${ }^{15}$ thyrotoxicosis might be induced. It should also be borne in mind that, in theory at least, the dose of thyroxine required might vary from time to time in individual patients as the presence and titre of TSH receptor antibodies fluctuates.

\section{References}

1 Slingerland, D W, et al, fournal of Clinical Endocrinology and Metabolism, $1972,35,912$.

2 Toft, A D, et al, British Medical fournal, 1974, 3, 152.

3 Seth, J, et al, Clinical Chimica Acta, 1976, 68, 291.

4 Irvine, W J, et al, Clinical Endocrinology, 1973, 2, 135.

${ }^{5}$ Toft, A D, et al, New England Fournal of Medicine, 1978, 298, 643.

6 Tunbridge, W M G, et al, British Medical fournal, 1974, 3, 89.

7 Toft, A D, et al, Lancet, 1975, 2, 576.

${ }^{8}$ Hedley, A J, et al, British Medical fournal, 1970, 1, 556.

${ }^{9}$ Boyle, C M, British Medical fournal, 1974, 2, 490.

10 Wise, P H, et al, Lancet, 1975, 2, 1231.

11 Bastenie, P A, et al, Lancet, 1977, 2, 155.

12 Fowler, P B S, Proceedings of the Royal Society of Medicine, 1977, 70, 297.

$13 \mathrm{Al}-\mathrm{Khateeb}, \mathrm{S} \mathrm{F}$, et al, Fournal of Clinical and Laboratory Immunology, 1978, 1, 55.

${ }_{14}$ Mukhtar, E D, et al, Lancet, 1975, 1, 713.

15 Evered, D, et al, British Medical fournal, 1973, 3, 131.

(Accepted 18 August 1978)

\title{
The social toll of Crohn's disease
}

\author{
B G GAZZARD, H L PRICE, G W LIBBY, A M DAWSON
}

British Medical fournal, 1978, 2, 1117-1119

\section{Summary and conclusions}

Eighty-five outpatients with Crohn's disease who knew their diagnosis and that the disease was subject to relapse were interviewed. The personalities of the women as measured by the Eysenck personality questionnaire were similar to those of a control group, but the men were more neurotic and introverted. Most patients continued to live optimistic, useful lives; they felt well, continued to work, hoped that their disease would not deteriorate in the future, and often had an improved relationship with their spouse despite less frequent sexual intercourse.

We found that the successful adaptation of patients to this chronic disease was more closely related to their personality than to the activity or extent of the disease.

\section{Introduction}

Crohn's disease is a life-long disabling condition that often necessitates multiple operations and prolonged drug treatment.

\section{Westminster Hospital, London SW1P 2AP}

B G GAZZARD, MD, MRCP, consultant gastroenterologist

Scarborough General Hospital, Scarborough, Toronto, Canada

H L PRICE, MD, FRCPC, consultant gastroenterologist

St Bartholomew's Hospital, West Smithfield, London EC1A 7BE

G W LIBBY, DPM, MRCPSYCH, senior registrar, department of psychological medicine

A M DAWSON, MD, FRCP, consultant gastroenterologist
When told of the diagnosis many patients are anxious to know how the condition will affect their life. Although detailed longitudinal studies of the clinical course of Crohn's disease have been reported, little information is available about the effect of the disease on the social, marital, and sexual lives of the patients and on their financial problems. We therefore undertook a survey of these aspects of the lives of patients with long-standing disease.

\section{Patients and methods}

All patients who had suffered from Crohn's disease for more than one year and attended the medical gastroenterology clinic between July and December 1976 were interviewed by a doctor who already knew them well, and enough time was given for them to be able to express themselves freely and at length. A form with questions about their knowledge of the disease, work record, married and sexual life, and financial problems was filled in for each patient. They were also asked a series of general questions about their ambitions and worries and how they saw their future. All patients completed a form to estimate their morbid anxiety index ${ }^{1}$ (MAI) and an Eysenck personality questionnaire $^{2}$ (EPQ). In calculating the MAI we excluded the question relating to bowel action and frequency. The degree of activity of Crohn's disease (disease activity) was assessed by a combination of clinical and biochemical features that we have previously found useful. While the potential maximum score on this index is 46 , most patients scoring above 10 have extensive active disease. ${ }^{3}$

Statistical analyses were performed using the Spearman ranking correlation coefficient unless otherwise stated.

\section{Results}

The 85 patients (28 male and 57 female) were aged 14-71 (mean 35.6 ) years. The duration of illness varied from one to 22 (mean 11) years. Over half the patients had disease in both the large and small 
intestines, and out of 50 who had undergone intestinal resection, 28 had been operated on on two or more occasions. Fifteen patients had an ileostomy or colostomy, and 66 were receiving immunosuppressive treatment when interviewed. Only four had not received these drugs or undergone surgery. Eight patients had a disease activity index of $8-12,32$ of $4-8$, and 45 of $0-4$ (10 patients had zero scores). The degree of disease activity bore no relation to any of the personality traits measured with the EPQ. The 24 patients who had been referred to us from other consultants had a similar disease activity index to that of the other patients in the series.

All the patients knew the correct name of their disease, and only three could not state which drugs they were taking. Only five patients, however, four of whom were nurses, appreciated the potentially serious side effects of drug treatment. Their descriptions of the nature of the disease were similar, as all but two said it was an inflammatory or fibrous lesion of the intestine that was subject to relapse and remission. Thirty-five of the patients had read about Crohn's disease. They usually had considerable difficulty in getting information, and the technical language made it impossible to understand. The four nurses and six university graduates found the information they had obtained themselves "helpful" and "reassuring," but it did not benefit the others. Two patients who read about Crohn's disease in the newspapers thought that it was a form of cancer. Sixteen patients volunteered that they would have liked to have had the opportunity to ask the doctor in more detail about their disease.

\section{MENTAL STATE}

In this series the personality of the women as measured by the EPQ was closely similar to that found in a large survey of normal controls, ${ }^{2}$ but the men were more introverted and neurotic. In a previous study the mean MAI of a group of normal controls of both sexes from which all patients with overt anxiety had been excluded was $7 \cdot 2 \pm$ SD 4 , no patient having a score of more than $14 .^{1}$ In our series 25 patients (10 male) had a MAI above 14 , and the mean score of all the patients was $13 \cdot 4 \pm 8$. The MAI and the neuroticism score measured on the EPQ were closely correlated $(P<0.001)$. Twentyeight patients had received or were receiving treatment for depression, and three other patients were thought to have severe personality disorders, including one with schizophrenia. There was a significant correlation between neuroticism score and treatment for depression but no relation between psychoticism and a presumed affective disorder. Patients referred to us from other hospitals tended to have a higher neuroticism score, but this failed to achieve statistical significance.

The patients' self-assessment of fitness indicated that 36 felt perfectly well and 38 fairly well. Surprisingly, only 11 patients felt poorly; most of these had high neuroticism scores (figure), while their disease activity index was similar to that of other patients in the series. Overall there was a significant correlation between the subjective well-being of the patient and the neuroticism and extraversion scores. Extraversion and neuroticism also correlated well with the patients' assessment of their prospects as happy, reasonable, or bleak (figure). Only 14 patients felt that their outlook was bleak, and nine of these were the patients who thought their disease would relapse in the future. They had high neuroticism scores, and most had been

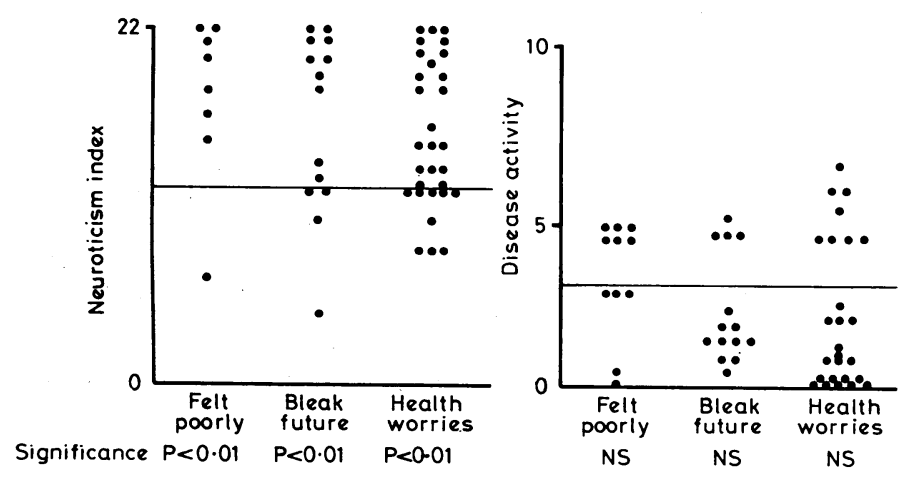

Neuroticism and disease activity scores for patients who felt poorly, thought they had a bleak future, and had health worries. Insufficient information was available to calculate neuroticism index for three patients who felt poorly and five who had health worries; disease activity could not be calculated for nine patients with health worries. Solid line represents mean value in whole series.

NS $=$ Not significant. treated for depression within the previous six months. Twenty patients felt their disease would stay the same, and 56 hoped it would improve.

Most patients mentioned domestic problems but not their health when listing their three main worries. The 35 patients who were worried about their health were more likely to be "neurotic" (figure), and 20 of them were being treated for depression when interviewed. Four of the women were concerned that the disease might affect their ability to have children. Patients with a diseased colon with or without an affected small intestine had more worries $\left(\chi^{2}=13.56 ; P<0.03\right)$, although these were not related to health.

\section{WORK RECORD AND FINANCES}

Thirty-eight of the 57 women worked regularly. Ten other women and one man worked part-time from choice. Only five patients had given up work completely despite long periods of absence through sickness. In the 22 patients who had had Crohn's disease for over two years there was no correlation between work record and either disease activity score at the time of interview or the number of operations performed. There was, however, a correlation between work record and MAI $(P=0.006)$. Only three patients felt that they could have achieved promotion or a better job through further training that had been denied them by ill health. Most patients were financially secure, only four needing to draw social security benefits or special invalid benefits.

All but four of the men had life insurance policies. Four patients had obtained cover before the Crohn's disease started, four obtained life insurance with no difficulty after the start of their disease, and the rest either had premiums which were loaded from $8 \%$ to $30 \%$ or were advised to take out short-term endowment policies.

\section{MARRIAGE AND FAMILY LIFE}

Three of the patients who were divorced thought that this was unrelated to their illness. Nineteen of the married patients considered that their marriage had improved because of their disease, as they had arrived at a more intimate understanding. This was particularly true of the extraverts, whereas those with a high neuroticism score felt their marriage relationship was worse or unchanged. Only nine patients thought that their marriage had suffered, and all of these had severe sexual problems. Out of the 52 patients who had married before developing Crohn's disease, 28 (15 women and 13 men) said that sexual intercourse had become less frequent, and a further 12 had ceased sexual contact altogether. Only 12 patients said that their sexual drive and frequency of intercourse was unaltered. The correlation between a worsening marital relationship and a decreased frequency of sexual intercourse was highly significant $\left(\chi^{2}=9.9 ; \mathrm{P}<0.01\right)$.

\section{Discussion}

Only patients who had suffered from Crohn's disease for more o than one year were included in the study to allow them enough time to gain some sort of perspective on their condition. Inpatients were excluded as their view of life was likely to be disturbed. Most of the patients, however, had been in hospital N during the preceding 12 months and had active disease treated $O$ by immunosuppressive treatment or surgery. The series may have been slanted towards patients who were more ill, since asymptomatic patients were reviewed only annually. As this study was not designed to investigate the pathogenesis of Crohn's disease it was not considered necessary to apply the personality questionnaire to a large group of controls. Interestingly, however, whereas the women in our series had similar personalities measured on the EPQ to those of people in a large control study, the men were more likely to be neurotic. The simplest explanation for this is that it is neurotic men who are willing to miss work to attend an outpatient clinic. In a previous study patients with Crohn's disease were more neurotic and introverted than chronic medical patients, but because of the small numbers it was not possible to determine whether this applied to both men and women. ${ }^{4} \mathrm{We}$ were unable to say whether the MAI of our patients differed significantly from normal as the 
results of this test on a normal population have apparently not been reported in detail.

The incidence of treatment for depression in these patients corresponded closely with that found in other series ${ }^{5}$; such treatment is most commonly given to neurotic patients, who appear to be more concerned about the course of their disease.

A startling finding was the number of patients who felt that their illness had improved their marital relationship. It was a recurrent theme that illness had drawn them closer together and increased mutual understanding. Sexual activity was a noticeable exception to this. Diminished sex drive has also been recorded in other chronic illnesses. ${ }^{6}$ In a study of Crohn's disease Ford et al found no major sexual problems but noted that their patients were "comparatively uninterested in sexual relationships." ; Many of our patients were unable to relax because of the fear of diarrhoea or abdominal pain, and in those marriages that had become less happy sexual dissatisfaction was nearly always the root cause.

Crohn's disease is a frightening diagnosis for both patient and doctor, with the prospect of chronic illness and acute exacerbations over many years and a death rate above that of the general population. ${ }^{8}$ Obviously the patient experiences considerable apprehension about his role in the family and at work, and the possibility of death. Thus interpersonal relationships will change at a time when the patient most needs them. Under these circumstances some degree of psychological regression is common, and patients with a poor premorbid psychological make-up-that is, who are neurotic-are likely to adapt badly.

This survey shows that the most important determinant of a patient's prospects is not the extent or activity of his disease but his personality, and underlines the truth of the old medical aphorism that the whole patient should be treated rather than the disease alone. Furthermore, it highlights some of the difficulties in using symptoms to assess disease activity when comparing the efficiency of various treatment regimens.

\section{References}

${ }^{1}$ Salkind, M R, Postgraduate Medical fournal, 1972, 5, 34.

2 Eysenck, H G, and Eysenck, S B G, Manual of the Eysenck Personality Questionnaire. London, Hodder and Stoughton, 1977.

3 Willoughby, J M T, et al, Lancet, 1971, 2, 944.

${ }^{4}$ Sheffield, B F, and Carney, M W P, British fournal of Psychiatry, 1976, 128, 446.

5 Whybrow, P C, and Ferrell, R B, in Emotional Factors in Gastrointestinal Illness, ed A E Lindner, p 82. Amsterdam, Excerpta Medica Foundation, 1973.

${ }^{6}$ Levy, N B, and Wynbrandt, G D, Lancet, 1975, 1, 1328.

7 Ford, C V, Glober, G A, and Castelnuovo-Tedesco, P, fournal of the American Medical Association, 1969, 208, 311.

8 Truelove, S G, and Pena, A S, Gut, 1976, 17, 192.

\title{
Respiratory distress syndrome in infants of Cardiff residents during 1965-75
}

\author{
IAIN CHALMERS, M E DAUNCEY, E R VERRIER-JONES, J A DODGE, O P GRAY
}

British Medical fournal, 1978, 2, 1119-1121

\section{Summary and conclusions}

The incidence of respiratory distress syndrome (RDS) among singleton infants of Cardiff residents was greater during 1970-4 than in the preceding five years. This was consistent with changes in the distribution of gestational age and birth weight. Case fatality rates among infants with RDS fell only slightly during the period examined.

Detailed examination of secular trends during 1965-75 suggested $(a)$ that increased use of elective delivery without assessment of pulmonary maturity increases the risk of RDS, and (b) that innovations in the management of RDS during the early 1970s cannot be assumed to have had widespread impact on case fatality rates.

\section{Introduction}

Respiratory distress syndrome (RDS) remains a major cause of neonatal morbidity ${ }^{1}$ and mortality ${ }^{2}$ despite developments in its

Welsh National School of Medicine, Cardiff CF4 4XN

IAIN CHALMERS, DCH, MRCOG, MRC research fellow, department of medical statistics (present address: National Perinatal Epidemiology Unit, Research Institute, Churchill Hospital, Oxford OX3 7LJ)

M E DAUNCEY, BA, computer programmer, department of medical statistics

E R VERRIER-JONES, DCH, FRCPED, consultant paediatrician

J A DODGE, MD, FRCPED, reader in child health

O P GRAY, DCH, FRCP, professor of child health prevention ${ }^{3-5}$ and treatment. ${ }^{6}$ In assessing the incidence of the syndrome, however, several difficulties arise. The first is in definition: RDS is a clinical diagnosis that may subsume one or more various pathological states, some of which may be confirmed only by necropsy. Although neonatal paediatricians in Switzerland ${ }^{1}$ and Sweden ${ }^{7}$ have agreed on diagnostic criteria for incidence studies, the diagnosis of RDS elsewhere continues to imply different things to different people. An additional problem is the lack of data on total populations of neonates. Most published studies refer to hospital case loads and may therefore distort true incidence rates. We know of only two published incidence studies referring to total populations of neonates, ${ }^{18}$ and only one of these examined secular trends in the incidence of RDS. ${ }^{8}$

Knowing current trends in the incidence of RDS seems important in view of the increasing number of reports of iatrogenic RDS after elective delivery. ${ }^{9-12}$ We decided to examine the incidence of RDS in Cardiff after observing that birth weight and gestational age distributions had "shifted to the left" during 1965-74. ${ }^{13}$ We were unable to explain this phenomenon by changes in maternal age, parity, height, smoking habits, or history of abortion and think that it may have resulted from increased use of elective delivery during 1969-72. We report here changes in the incidence of RDS and neonatal mortality rates attributed to the syndrome among infants born to Cardiff residents between 1965 and 1975.

\section{Materials and methods}

Since 1965 the Cardiff Births Survey has provided perinatal data on all deliveries to Cardiff residents regardless of where delivery takes place. All infants are followed up until the 28th day after birth, 